\title{
Biomechanic and histologic analysis of fibroblastic effects of tendon-to-bone healing by transforming growth factor $\beta 1$ (TGF- $\beta 1$ ) in rotator cuff tears ${ }^{1}$
}

\author{
Chong Zhang', Yu-Jie Liu"
}

'MD, Associated Professor, Department of Orthopaedic Surgery, the Chinese PLA General Hospital, Beijing, China.Intellectual and scientific content of the study, aquisition of data, statistical analysis, manuscript writing. "MD, Professor, Department of Orthopaedic Surgery, the Chinese PLA General Hospital, Beijing, China.Design, intellectual and scientific content of the study, critical revision.

\begin{abstract}
Purpose: To evaluate the effect of transforming growth factor $\beta_{1}$ (TGF- $\beta_{1}$ ) on tendon-to-bone reconstruction of rotator cuff tears.

Methods: Seventy-two rat supraspinatus tendons were transected and reconstructed in situ. At 8 and 16 weeks, specimens of three groups; that is control, L-dose (low dose), and $\mathrm{H}$-dose (high dose) were harvested and underwent a biomechanical test to evaluate the maximum load and stiffness values. Histology sections of the tendon-to-bone interface were identified by hematoxylin-eosin or Masson trichrome stain. Collagen type III was observed by picric acid sirius red staining under polarized light. The level of insulin-like growth factor 1 (IGF-1) and vascular endothelial growth factor (VEGF) was measured by the enzyme-linked immunosorbent assay (ELISA) method.
\end{abstract}

Results: Collagen type III of the H-dose group had a significant difference in histology structure compared with the $\mathrm{L}$-dose group $(\mathrm{P}<0.05)$. The maximum load and stiffness decreased significantly in the control group compared with the values of the L-dose and H-dose groups. The stiffness among the three groups differed significantly at the same postoperative time $(\mathrm{P}<0.05)$. Interestingly, progressive reestablishment of collagen type III affected tendon-tobone healing significantly in the later stages.

Conclusion: The $\mathrm{H}$-dose was associated with an increased collagen type III morphology stimulated by TGF- $\beta_{1}$.

Key words: Transforming Growth Factor beta 1. Tendons. Collagen Type III. Insulin-Like Growth Factor I. Vascular Endothelial Growth Factor. Rats. 


\section{- Introduction}

Rotator cuff tears (RCT) are a common cause of debilitating pain and compromised shoulder function. The rotator cuff tendon is damaged by friction, leading to the extrusion of the head of the humerus, acromion, and coracoacromial ligament through the shoulder joint, resulting in the weakening of the anatomical foundation. Numerous studies have clinically validated this type of impingement ${ }^{1,2}$. These injuries are compounded by tendon-tobone compression, which allows greater stress to be gradually delivered from the tendon to the cartilage-bone, or to gradually pass from the bone to the tendon, possibly causing tendon rupture ${ }^{3,4}$. There have been several studies in the literature reporting the complications of biological scaffolds ${ }^{5,6}$. Combinations of cell degeneration and necrosis, fiber degeneration, accumulation of calcification proteins such as collagen, and disappearance of the original wavy shape and small artery intimal hyperplasia may occur ${ }^{7}$. Hernigou et al. ${ }^{8}$ have reported that scaffold systems release biologically active insulin-like growth factor over mechanically robust slowly degrading scaffolds. Wardale et al..$^{9,10}$ demonstrated biologically improved rotator cuff healing in an elderly rat model using mesenchymal stem cells (MSCs) compared with other current techniques. Implantation of MSCs along with collagen type I scaffold was safe and effective for the healing of tendon defects. Thus, novel biological strategies to mechanically augment rotator cuff repair and enhance healing have great potential.

We hypothesized that transforming growth factor $\beta_{1}$ (TGF- $\beta_{1}$ ) sutured into the rat supraspinatus tendon-to-bone interface would increase the muscle's biomechanical strength and histologic organization, allowing for improved tendon-to-bone healing at the rotator cuff footprint.

\section{Methods}

This study was carried out in strict accordance with the recommendations in the Guide for the Care and Use of Laboratory Animals of the National Institutes of Health. The animal use protocol has been reviewed and approved by the Institutional Animal Care and Use Committee (IACUC) of the Chinese PLA General Hospital.

Seventy-two male Sprague-Dawley rats over 70 days of age with a mean preoperative body weight at surgery of $320 \pm 52.5 \mathrm{~g}$ were randomly divided into three groups (24 rats per group). TGF- $\beta 1$ was prepared for the animals in each group: the control group received no TGF- $\beta 1$ (control, surgical suture repair without additional intervention); the second group received a low concentration of TGF- $\beta 1$ (L-dose group, the sutured conjunction was mixed with a TGF- $\beta 1$ concentration treatment of 5.0 $\mathrm{ng} / \mathrm{mL}$ ); and the third group received a high concentration of TGF- $\beta 1$ (H-dose group, the sutured conjunction was mixed with a TGF- $\beta 1$ concentration treatment of $10.0 \mathrm{ng} / \mathrm{mL}$ ). The mixture was prepared so that each rat would receive a standard of $0.5 \mathrm{~mL}$ per $100 \mathrm{~g}$ weight. All animals were obtained from Beijing Vital River Laboratory Animal Technology Co., Ltd, China. The animals had free access to food and water in a pathogen-free animal room of ambient temperature $\left(20^{\circ} \mathrm{C}-23^{\circ} \mathrm{C}\right)$ and relative humidity (50\%-55\%). The feed composition was as follows: $40 \%$ corn meal, $15 \%$ wheat flour, $30 \%$ wheat bran, $5 \%$ yeast extract, and $10 \%$ water. All authors were blinded to the three groups and time intervals of the specimen testing.

Preparation of TGF-B

TGF- $\beta 1$ antibody (SC-146, $200 \mu \mathrm{g} / \mathrm{mL}$, $1.0 \mathrm{~mL} /$ bottle, Santa Cruz Biotechnology Inc., US) and fibrin glue (FG, $2.0 \mu \mathrm{g} / \mathrm{mL}, 2.5 \mathrm{~mL}$ / 
bottle, Shanghai Generay Biotech Co., Ltd, China) were prepared for the experiment. The TGF- $\beta 1$ antibody was added into high-pressure steam sterilized phosphate buffer saline $(0.5$ $\mu \mathrm{g} / \mathrm{mL})$, and then mixed with the solution of fibrin glue under aseptic conditions (5.0; 10.0 $\mathrm{ng} / \mathrm{mL}$ ). The animals' TGF- $\beta 1$ concentration level was divided into two groups as follows: low dose (TGF- $\beta 1$ concentration treatment of 5.0 $\mathrm{ng} / \mathrm{mL}$ ) and high dose (TGF- $\beta 1$ concentration treatment of $10.0 \mathrm{ng} / \mathrm{mL}$ ). Surgery with TGF- $\beta 1$ plus FG was performed to preserve TGF- $\beta 1$ high porosity in the three-dimensional structure.

\section{Surgical procedure}

An injury rat RCT model was established by surgically transecting the right forelimb supraspinatus tendons $(2.3 \mathrm{~mm})$ completely and repairing with a technique on the basis of a well-established protocol ${ }^{11}$.

We administered a kaloven injection (5\%, batch number 20110926, Kang Dien animal pharmaceutical Co., Ltd., Qingdao Shandong, China) according to $0.1 \mathrm{~mL} / \mathrm{kg}$ weight 0.5 to 1 hour before surgery to control postoperative pain for a period of 3 postoperative days. Ceftriaxone $(0.3 \mathrm{~g})$ was injected intramuscularly 30 minutes before surgery to prevent infection. All animals were anesthetized through an intraperitoneal injection of $3 \%$ sodium pentobarbital (16-20 mg/kg) before surgery. The first injection was $50 \%$ to $75 \%$ of the total amount of pentobarbital, followed by an additional $10 \%$ to $15 \%$ until the animal was successfully anesthetized.

The rat was placed in the lateral decubitus position. After shaving hair off the surgical site and sterilizing the area, a 2 to 2.5 $\mathrm{cm}$ longitudinal incision of the right shoulder by an open deltoid-splitting approach was made to expose the supraspinatus of the rotator cuff on the humerus greater tuberosity. The supraspinatus tendon was transected completely and released from the footprint.
The suture ends from the supraspinatus were passed through the bone tunnels to ensure tendon-to-bone postoperative healing. All right shoulders were allowed free movement when the animals were returned to their cages. Dual flush joint cavity suction drainage along the closed wounds was performed to ensure a lack of bleeding, and wounds were checked every other day for redness, swelling, and exudation. All rats were randomly divided into two groups for euthanization with deep anesthesia with an intravenous overdose of pentobarbital $(80 \mathrm{mg} /$ $\mathrm{kg}$ ) at 8 and 16 weeks postoperatively. Within each group of 24 rats, 12 rats were used for the mechanical test, and the other 12 rats underwent histologic analysis.

Specimens of the supraspinatus and proximal part of the humerus were collected for biomechanical testing and histologic analysis ( $n=6$ in each group at 8 and 16 weeks each). Specimens 3.0 to $3.5 \mathrm{~cm}$ in length were taken from the right forelimb to the large tubercle of the humerus, with the tail of the supraspinatus as the center of the two ends. All authors were blinded to the study groups and time intervals of the specimens at the time of the analysis.

\section{Histological analysis}

Specimens were sectioned parallel or perpendicular to the sagittal axis of the complexes and consisted of tendon-tobone interface at a thickness of $6 \mu \mathrm{m}$. All supraspinatus tendons were well attached, and no migration or gross infection was found in any of the TGF- $\beta 1$ complexes. To detect the situation in the living tissue, we removed the fresh specimen immediately and worked with frozen machine slices, rather than paraffin slices. The samples were fixed in $10 \%$ formalin and decalcified in methy-methacrylate compound, the conventional tissue treatment. Growth healing of the interface was observed by means of hematoxylin and eosin (H\&E) and Masson trichrome staining analysis performed 
by light microscopy (Olympus Co., Osaka, Japan) at 8 and 16 weeks vascularity, tendon fiber arrangement, and structure variations were analyzed. Accurate evaluations were assessed according to Longo et al. ${ }^{12}$.

Collagen fiber types were obtained by picric acid sirius red staining under polarized light. Growth of collagen fibers remodeling tissue and the quantity of collagen type I, II, and III were observed by a high-resolution image using a slide fluorescence microscope equipped with cameras (Leica DM4000-B, Germany). The primary color factors were observed for each group.

\section{Tendon-to-bone chemistry}

At 8 and 16 weeks, the content of IGF1 , determined by vascular endothelial growth factor (VEGF) from interfacial samples between the host-bone-and-graft complex were harvested for enzyme-linked immunosorbent assay (ELISA) analysis $(n=6)$. According to the manufacturer's protocol, the content of insulin like growth factor-1 (IGF-1) was performed with a rat IGF-1 ELISA kit (ab119548, Abcam Co., Ltd., Cambridge, USA). VEGF expression was detected by rat VEGF ELISA kit (ab100784, Abcam Co., Ltd., Cambridge, USA).

\section{Biomechanical testing}

The fresh supraspinatus tendons kept moist with normal saline were prepared for mechanical testing (8874, Instron, MA, USA) at room temperature and wax sealed immediately without being frozen. The biomechanical protocol was approved by a well-established model for detecting rat rotator cuff repair ${ }^{13-16}$.

The specimen was fixed on the machine and immediately clamped at $90^{\circ}$ with a screw (Figure 1). The specimen was then stressrelaxed at $5 \%$ strain for 5 minutes. Each test was performed by axially preloading to 0.10
$\mathrm{N}$ and a pretreatment tensile speed of 0.2 $\mathrm{mm} / \mathrm{s}$ for five cycles of preconditioning, and then applied at a $2.5 \mathrm{~N}$ loading measurement with an elongation speed of $0.6 \mathrm{~mm} / \mathrm{min}$ until broken down completely. The maximum load $(\mathrm{N})$ and stiffness value $(\mathrm{N} / \mathrm{mm})$ were recorded using Sigma Plot 9.0 (SPSS Inc., Chicago, IL, USA).

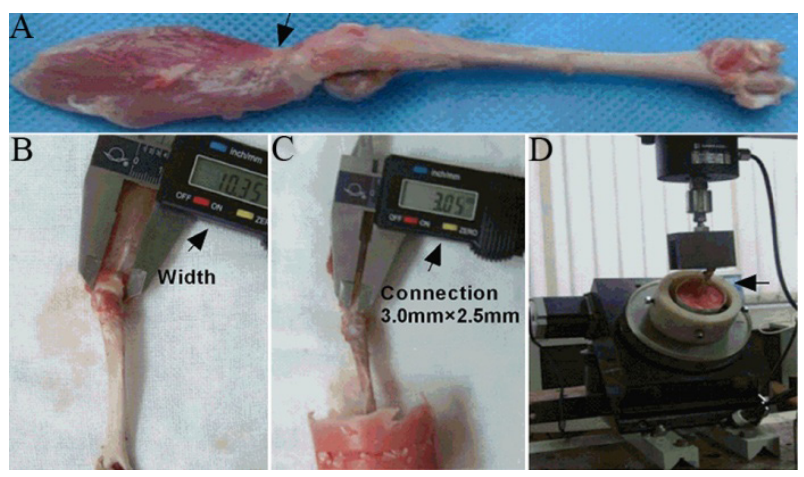

Figure 1 - Specimen of supraspinatus fixed in the mold of experiment for biomechanic test.

\section{Statistical analysis}

Data are presented as mean values \pm standard deviations (SD). All statistical analyses were performed using SPSS17.0 software (Jande, SPSS Science, Chicago, IL, USA) on a personal computer. The maximum load and tensile stiffness were compared with normal distribution and homogeneity by using factorial analysis of variance (ANOVA). Individual differences between the research and control groups at the same time point were delineated with a least significant difference $t$ test. Differences between groups at each time point were analyzed by using one-way ANOVA. Comparisons of the two experimental means were performed with a rank variance test. An independent t test was used to compare data of the same group at various time points. 
Semi-quantitative analyses of gray value were confirmed by imaging. The $P$ values were two sided, and the level of statistical significance was assumed at $\mathrm{P}<0.05$.

\section{- Results}

\section{H\&E staining}

Assessment of H\&E staining indicated significant differences at 8 and 16 weeks postoperatively in the three groups (Figure 2A). Briefly, the H-dose group was significantly different compared to the other two groups.

Fresh vascular proliferation and cartilage orderly arrangement were observed in all groups at this time point. There was less cellular and early cartilage formation in the L-dose group and matrix organization in line with the tendon of the control group (Figure 2AE). Fiber vascular granulation tissue remodeling and new hyaline cartilage were obviously visible at the tendon-to bone interface of the $\mathrm{H}$-dose group at 8 weeks (Figure $2 \mathrm{~A}-\mathrm{C}$ ). Tendon fibroblasts had formed progressively, with pale blue hyaline cartilage and cartilage-like cells increasingly oriented in bands in the specimen of the $\mathrm{H}$-dose group at 16 weeks (Figure $2 \mathrm{~A}-\mathrm{F}$ ).

\section{Masson trichrome stain}

As shown in Figure 2B (at 8 weeks), the tendon-to-bone interface cartilage fiber in control group showed significantly lower format orientation of beginning restoration compared to that of the L-dose group (Figure $2 \mathrm{Bb})$. Collagen fiber generation in the $\mathrm{H}$-dose group at 16 weeks was clearly seen in the middle of the images, especially forming a new chondrocyte point (Figure 2B-C). Suture proliferation of cartilaginous cells of $\mathrm{H}$-dose is seen in the top left corner of the image (Figure $2 \mathrm{BC}-\mathrm{f}$ ). The tendon-bone interface of the $\mathrm{H}$-dose cellular collagen and muscle remodeling is arranged in an orderly way compared with that of the other two groups.

\section{Picric acid sirius red staining}

The immunohistological analysis of collagen type III under polarized light microscopy showed significant differences among the three groups postoperatively. As shown in Figure $2 \mathrm{C}$ at 8 weeks, the collagen type II demonstrating red and bright white in the control group is quite noticeable in the middle of the image compared to the $\mathrm{H}$-dose images, in which the bright white appears in more and more areas at 16 weeks (Figure 2Ca, d). The L-dose group has a $39.5 \%$ proportion of yellow grains indicating collagen type $\mathrm{I}$, and a small amount of red collagen type II displayed in a relatively disordered arrangement compared with the other two groups (Figure $2 \mathrm{Cb}, \mathrm{e}$ ). Obvious green particles of collagen type III are closely arranged in a cluster in the $\mathrm{H}$-dose group were observed at 8 weeks (Figure 2Cc). To prolong the time-allowed activity of TGF- $\beta 1$, the supraspinatus tendon-to-bone interface at 16 weeks has dense orderly displayed wave conformation of the highly slim green collagen type III, which is arranged longitudinally of the tendon cells (Figure $2 \mathrm{Cf}$ ). The semi-quantitative scoring analysis of imaging shows relevant differences among the three groups (Figure 2D). Collagen type III proportion in the H-dose group is the maximum amount observed among the three groups. 

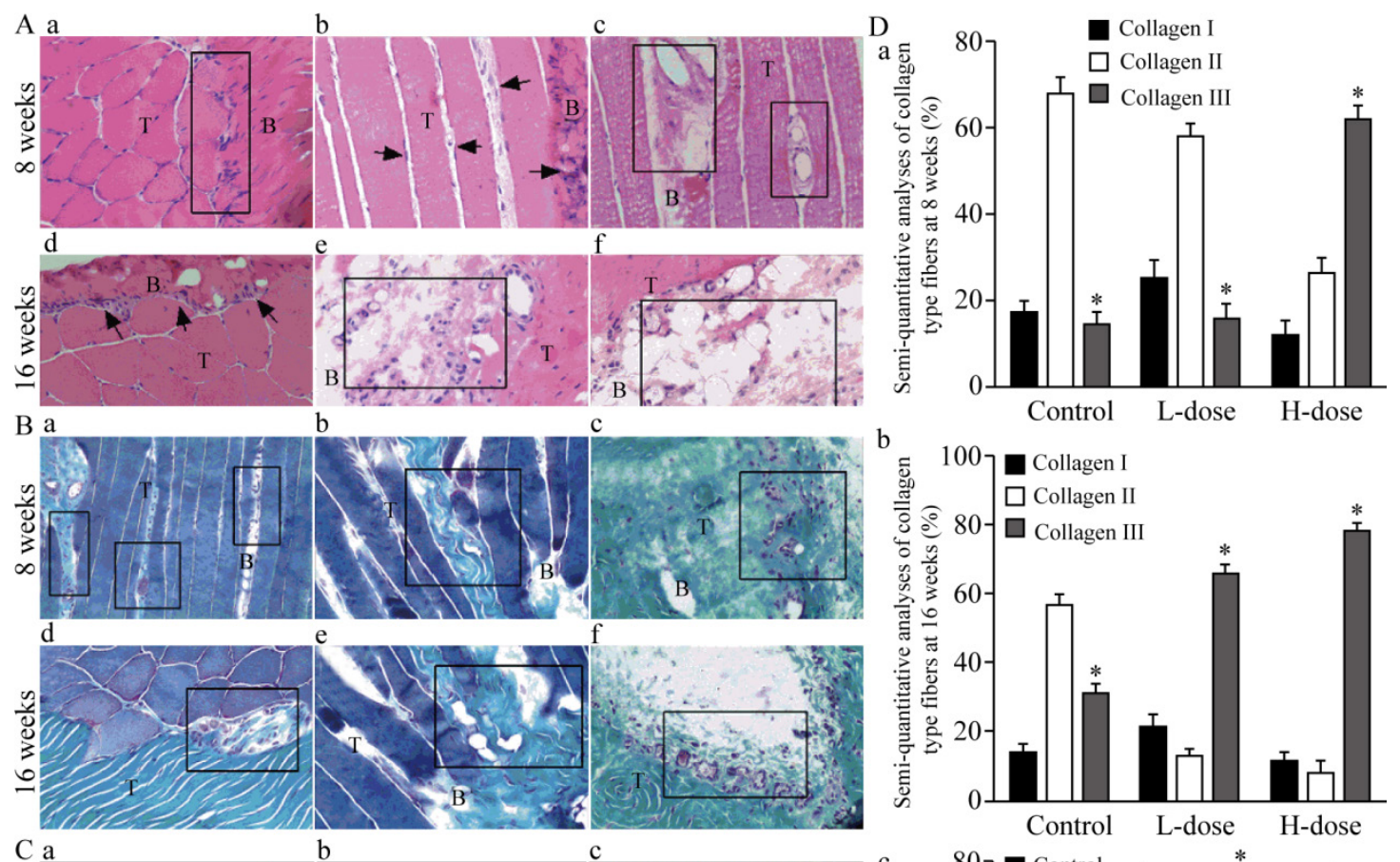

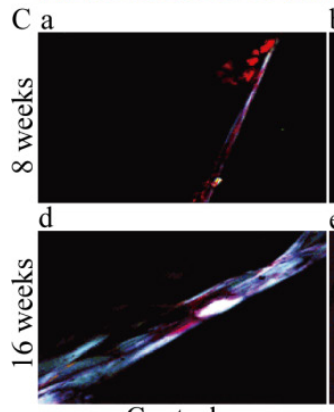

Control

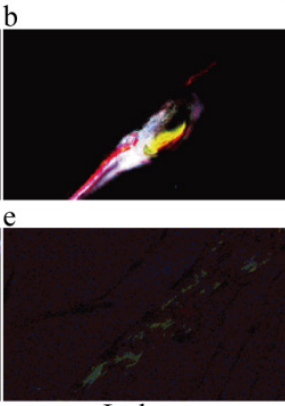

L-dose

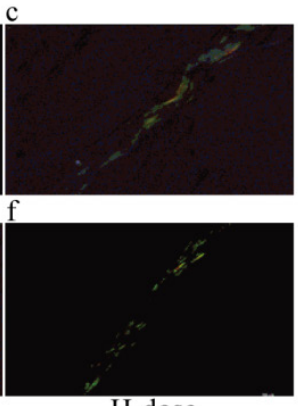

H-dose

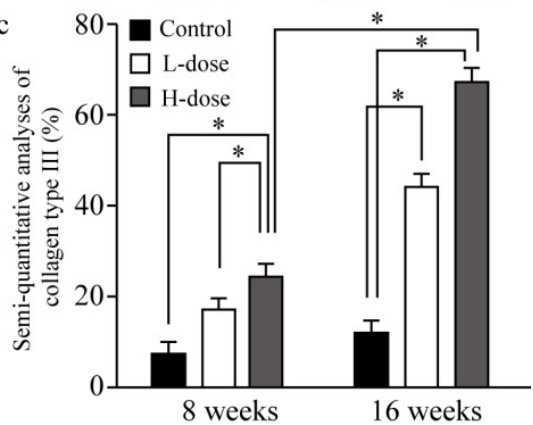

Figure 2 - Histological appearance of supraspinatus tendon-to-bone insertion with TGF- $\beta 1$ are shown. Hematoxylin and eosin staining; masson and picric acid-sirius red stain respectively. Arrows and rectangles show clusters of pale blue hyaline cartilage (Hematoxylin and eosin, $\times 80$ ). Rectangles indicate regions of interest blue black fibroblast nucleus cells (masson magnification, $\times 100$ ). Picric acid-sirius red staining magnification: $\times 40$ and bars correspond to $50 \mu \mathrm{m}$. $\mathrm{B}=$ bone; $\mathrm{T}=$ tendon.

\section{The level of IGF-1 and VEGF}

As shown in Figure 3 , the results of ELISA demonstrated that the application of TGF- $\beta 1$ improved significantly the expression of IGF-1 and VEGF after surgery $(P<0.05)$. At 8 and 16 weeks after surgery, the level of IGF-1 increased significantly in the $\mathrm{H}$-dose group compared with the control group $(\mathrm{P}<0.05)$. Interestingly, a trend toward a higher VEGF level in the $\mathrm{H}$-dose group was observed compared to the control and L-dose groups (Figure 3B). At 16 weeks after surgery, the H-dose group's tendon-to-bone content of VEGF data increased significantly compared with the other two groups $(P<0.05)$. 


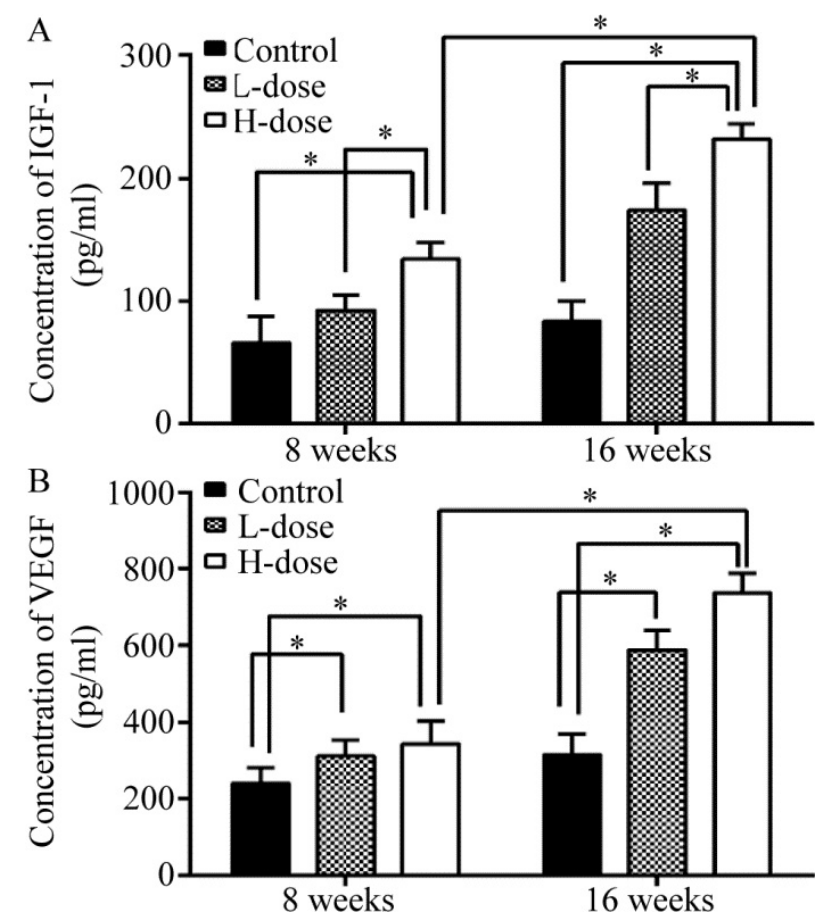

Figure 3 - Results of digitized image level of IGF1 and VEGF. Error bars correspond to standard deviations. ${ }^{*} \mathrm{P}<0.05$.

\section{Mechanical results}

All specimens failed at the tendon-tobone interface joint during the biomechanical test. As shown in Figure 4A, the maximum load of the $\mathrm{H}$-dose group supraspinatus tendon at 8 weeks postoperatively were stronger than those of the other two groups $(P<0.05)$. At 16 weeks, the increased maximum load to failure was significantly higher in the $\mathrm{H}$-dose group compared with the means of other two groups. TGF- $\beta 1$ in the control group was the weakest among the groups at both time points ( $F=$ $11.244, P=0.015, P<0.05)$. The final TGF- $\beta 1$ dose-time interaction showed an increasing trend of the maximum tensile strength ( $F=$ 9.207, $P=0.016, P<0.05)$. A statistical difference was observed in the tendon-to-bone injection of the three groups at the same time point.
As shown in Figure 4B, the maximaum stiffness of the $\mathrm{H}$-dose group increased significantly compared to data of the control group at 8 weeks $(F=11.005, P=0.001$, $P<0.05)$. The stiffness among the three groups was significantly different at the same time point postoperatively $(F=10.211, P=0.019$, $P<0.05)$. Differences of stiffness were observed between dose and time $(F=6.258, P=0.093$, $\mathrm{P}<0.05)$. Whereas the $\mathrm{H}$-dose group showed a significant difference compared with the means of the L-dose group at 8 and 16 weeks. At 16 weeks, shoulders that had not received TGF- $\beta 1$ (control group) were the least stiff compared with that of the L-dose group.

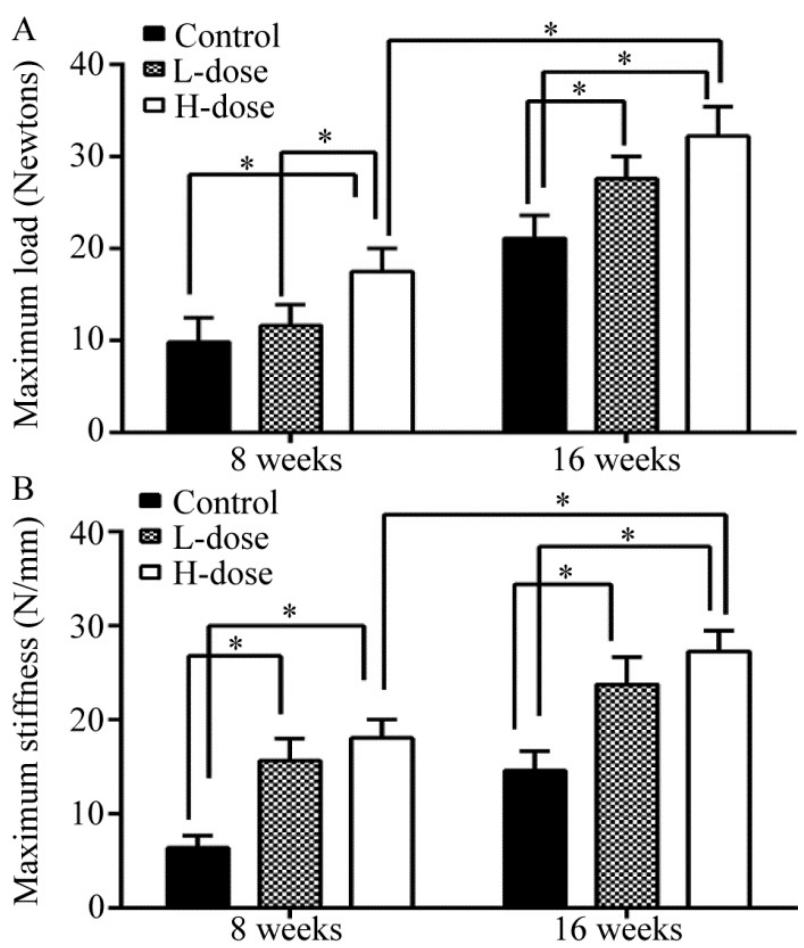

Figure 4 - Bar chart depicts biomechanic result as gray scales for each group after surgery. Error bars correspond to standard deviations. ${ }^{*} \mathrm{P}<0.05$.

\section{Discussion}

Clinical studies have shown a high rate of treatment after massive rotator cuff 
repair. Functional outcome after rotator cuff repair is important. We think it was imperative to determine the effect that TGF- $\beta 1$ has on physiological function and the healing process after rotator cuff repair. Our results have important implications for clinicians performing rotator cuff repair.

In this experiment, a sustainedrelease fibrin glue scaffold was used with TGF- $\beta 1$ to repair injured ligaments/tendons. In addition, proteins and increased collagen fiber contributed to enhancing mechanical properties of the supraspinatus construction. Similar to previous studies, Loiselle et al. ${ }^{17-21}$ performed antisense oligonucleotides (ASOs) injected into the tendon repair site at 2, 6, and 12 days post-surgery. Their results suggested that modulation of the TGF- $\beta 1$ signaling pathway could reduce adhesion while maintaining the strength of the repair. Alternatively, our study showed a positive effect of tendon-to-bone interface remodeling and a more rounded IGF-1 ossification occurring 16 weeks after surgery. Overall, our results suggested that administration of TGF- $\beta 1$ alters the loose connective tissue inflammatory process by increasing extra-cellular matrix homeostasis activity so as to accelerate healing. Klein et al. ${ }^{22-}$ ${ }^{23}$ demonstrated that macrophage infiltration and degeneration accompanied by increased IGF-1 and VEGF expression in a rat model of massive rotator cuff tears did not negatively affect outcomes. Compared to previous studies in our research, it was found that the rate of expression of IGF-1 and VEGF reached rapidity at 16 weeks, which may be at the peak of secretion.

TGF- $\beta 1$ molecules improve the formation of new fiber and fibrocartilage at the tendon-to-bone attachment site, resulting in improved load to failure. More importantly, the maximum load and stiffness were increased significantly in the H-dose group from 8 to 16 weeks compared with the other groups in our study. A similarfinding was described by Lamplot et $a .^{24}$, who investigated the upregulated fibronectin expression of bone morphogenetic protein 13 (BMP13, a growth factor of TGF). Results showed that BMP13 promoted tendon healing, because of its superior biological factor, and reduced the incidence of re-tearing. Hatta et al. ${ }^{25-29}$ demonstrated that BMP13 provided significantly better biomechanical performance when compared to traditional surgery in multiple double rows using the supraspinatus tendon in nine shoulders per group. In the current study, a greater degree of maximum load and stiffness at 16 weeks in the $\mathrm{H}$-dose group suggested differences that were significantly higher than those of the other two groups. Our biomechanical results confirmed that TGF- $\beta 1$ had higher intensity sustained-release expression of IGF1 and VEGF in the matrix. Collagen type III regenerated at the tendon interface because of the effect of the combination of IGF-1 and VEGF. As far as maximum load and stiffness, the tendon-to-tendon reconstruction enhanced the mechanism load of action and efficacy significantly in the supraspinatus tendon.

The development of tendon-tobone healing for stimulation of engineered constructs with biological activity for the treatment of rotator cuff tears has expanded rapidly over the past decades. In recent years, numerous studies have shown large failure rates, causing concern about supraspinatus strangulation contractures from adhesion by fatty infiltration. Chen et al..$^{30-32}$ investigated the effects of chitosan on Achilles tendon injury in rats 8 weeks after surgery. Gliding excursion and the content of collagen fibers were used to evaluate the effects of surgery. They demonstrated that chitosan improved the condition of scar tissue and glide tendon healing by reducing the high expression of miR-29b and the downregulation of TGF- $\beta 1 /$ Smad3 level. Conversely, the researchers found that the supraspinatus tendon-to-bone interface was arranged in a dense orderly 
waved formation similar to normal tendon morphology, and collagen type III facilitated a direct point of footprint area. Tsai et al. ${ }^{33,34}$ previously stated that an increase in TGF- $\beta$ stimulated the expression of collagen type III and $I$ in the healing process. The purpose of the study was to determine the expression of collagen type III in the rat Achilles tendon using ultrasound and to determine whether a correlation exists between the expression and promotion of synthesis of collagen in the intrinsic tendon cells when using TGF- $\beta 1$. Meanwhile, in our study, the tendon-to-bone healing has suggested that the amount of collagen type III significantly accelerated at 16 weeks as well as proliferated matrix synthesis and cartilage regeneration.

To the best of our knowledge, this is the first study to investigate the effect of TGF- $\beta 1$ on tendon bone healing. TGF- $\beta 1$ upgrades tendonto-bone-related growth factor expression and improves supraspinatus mechanical strength and maturity at the remodeled tendon-tobone junction. Results showed that TGF- $\beta 1$ promoted tendon cell proliferation synthesis by collagen type III by promoting tendonto-bone healing primarily. We also observed the tendon-to-bone healing at the junction because of the collagen type III-initiated growth. Although collagen type $I$ is the most abundant protein and a major component of the extra-cellular matrix, more importantly, collagen type III would be more extracellular membrane proliferation and the ability to synthesize collagen. It can initiate fibroblast growth that can be evaluated for healing factors and mechanical strength. According to the experiment statistics, IGF-1 and VEGF were expressed at a high level, which supported the collagen type III formation in the $\mathrm{H}$-dose group, $60 \%$ of which was found in the parenchyma at the tendon-to-bone interface.

In summary, microscopic observation in the three groups showed an obvious layer of fibroblasts tightly packed between the cartilage-like cells, as well as an acceleration of regeneration of collagen type III as opposed to an inhibition of inflammation and collagen fibers and a small amount of visible connection fibrocartilage (control group). Further studies on reticular and elastic fibers of tendon-tobone interface would help further our findings.

\section{Conclusions}

TGF- $\beta 1 Z$ of $\mathrm{H}$-dose group had positive effects on the tendon healing compared to other two groups in rat rotator cuff. Collagen fibers in the $\mathrm{H}$-dose group promoted the transformation of loose connective tissue to dense tendon-to-bone tissue. Higher concentrations of TGF- $\beta 1$ resulted in stronger load and stiffness values, and the strength was more than that found in the group that did not use TGF- $\beta 1$, demonstrating that TGF- $\beta 1$ is associated with the delivery of more collagen type III in tendon repair.

\section{- References}

1. Abtahi AM, Granger EK, Tashjian RZ. Factors affecting healing after arthroscopic rotator cuff repair. World J Orthop. 2015 Mar 18;6(2):211-20. PMID: 25793161.

2. Gartsman GM, Brinker MR, Khan M. Early effectiveness of arthroscopic repair for full-thichness tears of the rorator cuff:an outcome analysis. J Bone Joint Surg Am. 1998 Jan;80(1):33-40. PMID: 9469306.

3. Gwak HC, Kim CW, Kim JH, Choo HJ, Sagong SY, Shin J. Delaminated rotator cuff tear: extension of delamination and cuff integrity after arthroscopic rotator cuff repair. J Shoulder Elbow Surg. 2015 May;24(5):71926. PMID: 25457782.

4. Rhee YG, Cho NS, Yoo JH. Clinical outcome and repair integrity after rotator cuff repair in patients older than 70 years versus patients younger than 70 years. Arthroscopy. 2014 May;30(5):546-54. PMID: 24630958.

5. Gilotra M, Nguyen T, Christian M, Davis D, Henn RF, Hasan SA. Botulinum toxin is 
detrimental to repair of a chronic rotator cuff tear in a rabbit model. J Orthop Res. 2015 Aug;33(8):1152-7. PMID: 25626677.

6. Cho E, Zhang Y, Pruznak A, Kim HM. Effect of tamoxifen on fatty degeneration and atrophy of rotator cuff muscles in chronic rotator cuff tear: An animal model study. J Orthop Res. 2015 Dec;33(12):1846-53. PMID: 26121952.

7. Ratcliffe A, Butler DL, Dyment NA, Cagle PJ Jr, Proctor CS, Ratcliffe SS, Flatow EL. Scaffolds for tendon and ligament repair and regeneration. Ann Biomed Eng. 2015 Mar;43(3):819-31. PMID: 25650098.

8. Hernigou $P$, Merouse $G$, Duffiet $P$, Chevalier $\mathrm{N}$, Rouard $\mathrm{H}$. Reduced levels of mesenchymal stem cells at the tendon-tobone interface tuberosity in patients with symptomatic rotator cuff tear. Int Orthop. 2015 Jun;39(6):1219-25. PMID: 25757411.

9. Wardale J, Mullen L, Howard D, Ghose S, Rushton N. An ex vivo model using human osteoarthritic cartilage demonstrates the release of bioactive insulin-like growth factor-1 from a collagen glycosaminoglycan scaffold. Cell Biochem Funct. 2015 Jul;33(5):277-84. PMID: 26059711.

10.Tornero-Esteban P, Hoyas JA, Villafuertes $E$, Rodríguez-Bobada C, López-Gordillo Y, Rojo FJ, Guinea GV, Paleczny A, Lópiz-Morales Y, Rodriguez-Rodriguez L, Marco F, FernándezGutiérrez B. Efficacy of supraspinatus tendon repair using mesenchymal stem cells along with a collagen I scaffold. J Orthop Surg Res. 2015 Aug 14;10:124. PMID: 26268217.

11.Soslowsky LJ, Carpenter JE, DeBano CM, Banerji I, Moalli MR. Development and use of an animal model for investigations on rotator cuff disease. J Shoulder Elbow Surg. 1996 Sep-Oct;5(5):383-92. PMID: 8933461.

12.Longo UG, Franceschi $F$, Ruzzini L, Rabitti C, Morini S, Maffulli N, Denaro V. Characteristics at haematoxylin and eosin staining of ruptures of the long head of the biceps tendon. Br J Sports Med. 2009 Aug;43(8):603-7. PMID: 18070808.

13.Galatz LM, Charlton N, Das R, Kim HM, Havlioglu N, Thomopoulos S. Complete removal of load is detrimental to rotator cuff healing. J Shoulder Elbow Surg. 2009 Sep-Oct;18(5):669-75. PMID: 19427237.

14.Carpenter JE, Thomopoulos S, Flanagan CL, DeBano CM, Soslowsky LJ. Rotator cuff defect healing: a biomechanical and histologic analysis in an animal model. J Shoulder Elbow Surg. 1998 Nov-Dec;7(6):599-605. PMID: 9883420.

15. Thomopoulos S, Kim HM, Rothermich SY, Biederstadt C, Das R, Galatz LM. Decreased muscle loading delays maturation of the tendon enthesis duringpostnatal development. J Orthop Res. 2007 Sep;25(9):1154-63. PMID: 17506506.

16. Galatz LM, Silva MJ, Rothermich SY, Zaegel MA, Havlioglu N, Thomopoulos S. Nicotine delays tendon-to-bone healing in a rat shoulder model. J Bone Joint Surg Am. 2006 Sep;88(9):2027-34. PMID: 16951120.

17.Loiselle AE, Yukata K, Geary MB, Kondabolu S, Shi S, Jonason JH, Awad HA, O'Keefe RJ. Development of antisense oligonucleotide (ASO) technology against Tgf- $\beta$ signaling to prevent scarring during flexor tendon repair. J Orthop Res. 2015 Jun;33(6):859-66. PMID: 25761254.

18. Baums MH, Schminke B, Posmyk A, Miosge $\mathrm{N}$, Klinger HM, Lakemeier S. Effect of single and double-row rotator cuff repair at the tendon-to-bone interface: preliminary results using an in vivo sheep model. Arch Orthop Trauma Surg. 2015 Jan;135(1):1118. PMID: 25416099.

19.Andarawis-Puri N, Flatow EL, Soslowsky LJ. Tendon basic science: Development, repair, regeneration and healing. J Orthop Res. 2015 Jun;33(6):780-4. PMID: 25764524.

20. Thomopoulos S, Parks WC, Rifkin DB, Derwin KA. Mechanisms of tendon injury and repair. J Orthop Res. 2015 Jun;33(6):832-9. PMID: 25641114.

21.Zingman $A$, Li $H$, Sundem L, DeHority $B$, Geary M, Fussel T, Mooney R, Zuscik M, Elfar J. Shoulder arthritis secondary to rotator cuff tear: a reproducible murine model and histopathologic scoring system. J Orthop Res. 2017 Mar;35(3):506-514. PMID: 27500994.

22. Klein MB, Yalamanchi N, Pham H, Longaker MT, Chang J. Flexor tendon healing in vitro:effects of TGF-beta on tendon cell collagen production. J Hand Surg Am. 2002 Jul;27(4):615-20. PMID: 12132085.

23. Feng $R, M a X, M a J$, Jia $H, M a B, X u L$, Liu A. Positive effect of IGF-1 injection on gastrocnemius of rat during distraction osteogenesis. J Orthop Res. 2015 
Oct;33(10):1424-32. PMID: 25452218.

24.Lamplot JD, Angeline $M$, Angeles J, Beederman $M$, Wagner $E$, Rastegar F, Scott B, Skjong C, Mass D, Kang R, Ho S, Shi LL. Distinct effects of platelet-rich plasma and BMP13 on rotator cuff tendon injury healing in a rat model. Am J Sports Med. 2014 Dec;42(12):2877-87. PMID: 25193888.

25. Hatta T, Giambini H, Uehara K, Okamoto S, Chen S, Sperling JW, Itoi E, An KN. Quantitative assessment of rotator cuff muscle elasticity: Reliability and feasibility of shear wave elastography. J Biomech. 2015 Nov 5;48(14):3853-8. PMID: 26472309.

26.Smith MJ, Cook JL, Kuroki K, Jayabalan PS, Cook CR, Pfeiffer FM, Waters NP. Comparison of a novel bone-tendon allograft with a human dermis-derived patch for repair of chronic large rotator cuff tears using a canine model. Ultrason Sonochem. 2016 Jan;28:169-77. PMID: 26384896.

27.Islam A, Bohl MS, Tsai AG, Younesi M, Gillespie R, Akkus O. Biomechanical evaluation of a novel suturing scheme for grafting load-bearing collagen scaffolds for rotator cuff repair. Clin Biomech (Bristol, Avon). 2015 Aug;30(7):669-75. PMID: 26009492.

28.Ahmad CS, Kleweno C, Jacir AM, Bell JE, Gardner TR, Levine WN, Bigliani LU. Biomechanical performance of rotator cuff repairs with humeral rotation: a new rotator cuff repair failure model. Am J Sports Med. 2008 May;36(5):888-92. PMID: 18400947.

29.Davies MR, Lee L, Feeley BT, Kim HT, Liu $X$. Lysophosphatidic acid-induced RhoA signaling and prolonged macrophage infiltration worsens fibrosis and fatty

\section{Correspondence:}

Yu-Jie Liu

Department of Orthopaedic Surgery, the Chinese PLA General Hospital

№ 28 Fuxing Road, Haidian District

Beijing 100853 China

Phone: +86-010-66936853

czdoccn@163.com

Received: Aug 18, 2017

Review: Oct 16, 2017

Accepted: Nov 19, 2017 infiltration following rotator cuff tears. J Orthop Res. 2017 Jul;35(7):1539-47. PMID: 27505847.

30.Chen $Q$, Lu $H$, Yang $H$. Chitosan inhibits fibroblasts growth in Achilles tendon via TGF- $31 /$ Smad3 pathway by miR-29b. Int J Clin Exp Pathol. 2014 Dec 1;7(12):8462-70. PMID: 25674210.

31.Taniguchi N, Suenaga N, Oizumi N, Miyoshi $\mathrm{N}$, Yamaguchi $\mathrm{H}$, Inoue $\mathrm{K}$, Chosa $\mathrm{E}$. Bone marrow stimulation at the footprint of arthroscopic surface-holding repair advances cuff repair integrity. J Shoulder Elbow Surg. 2015 Jun;24(6):860-6. PMID: 25487905.

32. Meyer GA, Farris AL, Sato E, Gibbons M, Lane JG, Ward SR, Engler AJ. Muscle progenitor cell regenerative capacity in the torn rotator cuff. J Orthop Res. 2015 Mar;33(3):421-9. PMID: 25410765.

33.Tsai WC, Pang JH, Hsu CC, Chu NK, Lin MS, Hu CF. Ultrasound stimulation of types I and III collagen expression of tendon cell and upregulation of transforming growth factor beta. J Orthop Res. 2006 Jun;24(6):1310-6. PMID: 16705693.

34.Angeline ME, Ma R, Pascual-Garrido C, Voigt C, Deng XH, Warren RF, Rodeo SA. Effect of diet-induced vitamin $D$ deficiency on rotator cuff healing in a rat model. Am J Sports Med. 2014 Jan;42(1):27-34. PMID: 24131579.

\section{Acknowledgements}

To classmates of the Laboratory Animal Center, the Chinese PLA General Hospital, for their assistance.

Conflict of interest: none

Financial source: Hebei Province Science and

Technology Project (№ 152777171)
${ }^{1}$ Research performed at Department of Orthopaedic Surgery, Army Sports Medicine Laboratory, the Chinese PLA General Hospital, Beijing, China. 


\section{Erratum}

Manuscript: Biomechanic and histologic analysis of fibroblastic effects of tendon-to-bone healing by transforming growth factor $\beta 1$ (TGF- $\beta 1$ ) in rotator cuff tears

Publication: Acta Cir Bras. 2017;32(12):1045-1055.

DOI: http://dx.doi.org/10.1590/s0102-865020170120000006

\section{Where was it read:}

(I) Associated Professor, Department of Orthopaedic Surgery, Traditional Chinese Medicine Hospital, Hebei Province, China. Aquisition of data, manuscript writing.

(II) MD, Professor, Department of Orthopaedic Surgery, Chinese PLA General Hospital, Beijing, China. Design, intellectual and scientific content of the study; critical revision.

\section{Read:}

IMD, Associated Professor, Department of Orthopaedic Surgery, the Chinese PLA General Hospital, Beijing, China. Intellectual and scientific content of the study, aquisition of data, statistical analysis, manuscript writing

IIMD, Professor, Department of Orthopaedic Surgery, the Chinese PLA General Hospital, Beijing, China. Design, intellectual and scientific content of the study, critical revision. 